Rabaska

Revue d'ethnologie de l'Amérique française

\title{
Institut pour le patrimoine de la francophonie de l'Ouest canadien (Campus Saint-Jean)
}

\section{Urbain Yamb et Claude Couture}

Volume 16, 2018

URI : https://id.erudit.org/iderudit/1051377ar

DOI : https://doi.org/10.7202/1051377ar

Aller au sommaire du numéro

Éditeur(s)

Société québécoise d'ethnologie

ISSN

1703-7433 (imprimé)

1916-7350 (numérique)

Découvrir la revue

Citer ce document

Yamb, U. \& Couture, C. (2018). Institut pour le patrimoine de la francophonie de l'Ouest canadien (Campus Saint-Jean). Rabaska, 16, 357-359.

https://doi.org/10.7202/1051377ar d'utilisation que vous pouvez consulter en ligne. 
nouvelle anthologie, un projet de diffusion qui s'échelonnera sur plusieurs années. L'anthologie peut être consultée au site : atlas.cieq.ca/la-francophonie-nordamericaine/recits-de-voyage-et-de-migration-une-nouvelle-anthologie.html.

Enfin, la CRC-MTCF a organisé la cinquième conférence annuelle « RobertPainchaud » en mars 2018. Cette année le professeur-chercheur Jean Morency, de l'Université de Moncton, prononçait une conférence intitulée « Jack Kérouac, écrivain franco-américain. Entre rêve et cauchemar ». Dans le cadre du Mois de la francophonie, la CRC-MrCF organisait également, cette fois conjointement avec l'AcFAs-Manitoba, une table ronde sur les circulations impériales à laquelle participaient Claude Couture et Srilata Ravi (Campus Saint-Jean, Université d'Alberta) et Phi-Vân Nguyen (Université de Saint-Boniface).

Yves Frenette et Yves Labrèche

\section{Institut pour le patrimoine}

de la francophonie de l'Ouest canadien

Faculté Saint-Jean

Université d'Alberta

8406, rue Marie-Anne-Gaboury

Edmonton, Alberta

T6C 4G9

Téléphone : (780) 465-8700

Télécopieur : (780) 465-8760

Courriel : saintjean@ualberta.ca

Toile : www.ualberta.ca/campus-saint-jean

\section{Aperçu}

L'année 2017-2018 a été une année assez significative pour l'Institut du patrimoine de la francophonie de l'Ouest canadien (IPFOC). Les faits marquants de l'année ont été : la mise à jour du catalogue ; l'achat d'une nouvelle base de données pour la gestion améliorée de nos archives ; la migration des données d'archives vers la nouvelle base de données ; le début de numérisation de nos archives audiovisuelles ; la collaboration avec des chercheurs et enseignants pour la réalisation d'un projet lié aux études linguistiques francophones dans l'Ouest canadien. Sur le plan positif, l'Institut a entrepris des négociations afin d'intégrer ses archives avec celles des archives de l'Université de l'Alberta. Plusieurs rencontres avec les représentants des Bibliothèques de l'Université de l'Alberta ont abouti à la mise en place d'un document projet (Business Case) proposant les étapes à suivre, les contraintes et les avantages de ce processus d'intégration.

\section{Activités réalisées}

1. Cérémonie de nomination des salles du Campus Saint-Jean (15 septembre 2017). Le projet a consisté à faire graver des plaques historiques expliquant la contribution au rayonnement de la communauté francophone en Alberta des individus remarquables qui sont ainsi honorés : grand salon : salle Marcelle-et-Louis-Desrochers ; salle historique : salle Onésime-Dorval (1845-1932); salle du Conseil (304) : salle Gamila-Morcos ; auditorium : salle Gertrude-Laing (1905-2005) ; amphithéâtre et atelier d'écriture : salle Marguerite-Primeau (1914-2011). 
2. Colloque international « Autour de l'œuvre d'Yvan Lamonde. Colonialisme et modernité au Canada depuis 1867 » au Campus Saint-Jean et Lister Center (25-27 octobre 2017). Ce colloque a réuni une vingtaines de conférenciers et chercheurs venus de plusieurs universités canadiennes et françaises.

3. Professorat Louis-Desrochers : «Yvan Lamonde (20 novembre-2 décembre 2017). Les activités tournaient autour des interventions en classe : M. Lamonde a abordé le thème de la laïcité en relation avec la question nationale (cours de Frédéric Boily : ÉTCAN 360 - La Question nationale au Canada) ; la littérature des francophonies canadiennes et acadienne (cours de Valérie Lapointe-Gagnon : ÉTCAN 330 - Les Francophonies canadiennes et acadiennes I : perspectives historiques et culturelles); le Québec et la francophonie internationale (cours de Srilata Ravi : FrANC 330 - Introduction aux francophonies littéraires hors-Canada) ; le Québec et les littératures francocanadiennes (cours de Pamela Sing : Franc 324 - Introduction aux francophonies littéraires du Canada - par le biais des représentations des Autochtones). Durant des présentations du midi, il a aussi traité de ses livres (Louis-Antoine Dessaulles ; Un coin dans la mémoire; L'Hiver de notre mécontentement; et du féminisme dans un entretien autour des cinq intellectuelles contemporaines dont parlent le Dictionnaire des intellectuel(les) au Québec: France Théoret (n. 1942), Nicole Brossard (n. 1943), Monique Larue (n. 1948), Martine Delvaux (n. 1968) et Aurélie Lanctôt (n. 1991) : S'agirait-il de deux ou de trois générations ? Qu'est-ce que les deux (ou trois) « vagues » auraient en commun d'un côté et, de l'autre, de distinct ?)

4. Le Centre d'archives - Janvier 2018 : mise à jour du catalogue des archives SaintJean ; février 2018 : envoi des archives audiovisuelles à l'atelier de numérisation du campus principal ; mars 2018 : réalisation du premier exemplaire du document projet (Business Case) portant sur l'intégration des archives Saint-Jean aux archives de l'Université de l'Alberta en collaboration avec l'équipe dirigeante des Bibliothèques de l'Université de l'Alberta ; réception de deux disques durs d'archives audiovisuelles numérisées ; avril 2018: fin du projet (Business Case) et mise à la disposition de la hiérarchie ; mai 2018 : visite guidée du Research and Collections Resource Facility (RCRF) qui tiendra lieu de dépôt de toutes les archives de l'Université de l'Alberta et qui devra abriter dorénavant les collections des archives Saint-Jean.

5. La salle historique - supervision d'une dizaine de visites guidées de la salle historique (salle Onésime-Dorval) dont les plus marquantes sont respectivement la visite du personnel de la Faculty Relation of University of Alberta pour leur retraite; de nombreux groupes scolaires francophones et d'immersion de la ville d'Edmonton en l'occurrence l'école Mc Kernan, Hunting Hills High School Red Deer et Archbishop Jordan High School.

\section{Activités à réaliser pour l'automne 2018}

Approbation du document projet (Business Case) intégrant les archives Saint-Jean aux archives de l'Université de l'Alberta par les autorités compétentes (juillet-août). Début du processus d'intégration des archives Saint-Jean aux archives de l'Univer- 
sité de l'Alberta et dépôt des collections au RCRF (août-septembre). Ce vaste projet consiste à sécuriser les collections des archives Saint-Jean en les stockant dans un lieu sûr selon les normes internationales actuelles de protection des archives. Le RCRF, situé au campus sud de l'Université de l'Alberta et inauguré le 31 mai 2018, est l'endroit approprié pour abriter toutes nos archives.

Urbain Yamb et Claude Couture 